\title{
Leprosy transmission and mucosal immunity: towards eradication?
}

\author{
I. A. CREE* \& W. CAIRNS SMITH ${ }^{\dagger}$ \\ *Department of Pathology, Institute of Ophthalmology, University \\ College London, Bath Street, London ECIV 9EL, UK; \\ ${ }^{\dagger}$ Department of Public Health, University of Aberdeen Medical \\ School, Polwarth Building, Foresterhill, Aberdeen AB9 2ZD, UK
}

\section{Accepted for publication 13 November 1997}

\begin{abstract}
Summary The declining prevalence of leprosy has not been matched by a declining incidence. Widespread adoption of mutliple drug therapy (MDT) in closely monitored control programmes has not prevented transmission of Mycobacterium leprae. Despite the rarity of lepromatous patients, most of those living in endemic areas have immunological evidence of exposure to $M$. leprae. This paradox could be explained if, for many such individuals, infection was transient, did not result in disease development, but did allow the transmission of infection to other individuals. There is increasing evidence from nasal PCR studies that such sub-clinical transmission may exist and that mucosal immune responses to $M$. leprae may develop during resolution of initial infection. Sub-clinical infection appears to occur in clusters and may require close contact over a prolonged period for optimal transmission. Control of transmission may be feasible through identification and treatment of individuals within infection clusters, allowing progress towards the eradication of leprosy.
\end{abstract}

\section{Introduction}

Over the last decade, the use of multidrug therapy (MDT) has reduced the estimated world prevalence of leprosy from around 12 million in 1983 to approximately 1.3 million active cases now. ${ }^{1}$ This reduction prompted the World Health Organization to aim for 'elimination of leprosy as a public health problem', defined as reduction in prevalence below 1 per 10,000 population. ${ }^{1}$ While this may be achievable, leprosy is far from beaten. In some series, up to one-third of patients eventually develop disability due to nerve damage and over 7 million 'cured' cases may still require ongoing care. ${ }^{1}$ More seriously, in general, the declining prevalence has not been matched by a declining incidence, except in countries with significant economic development. Widespread use of MDT has not yet prevented continued transmission. $^{2}$ 


\section{Source of infection}

The transmission of leprosy is poorly understood, but infection from subclinical sources could be more important than infection from active clinically apparent cases. ${ }^{3-5}$ Nearly all individuals in highly leprosy-endemic areas have immunological evidence of exposure to Mycobacterium leprae ${ }^{6-8}$ despite the relative rarity in most populations of the lepromatous patients thought to be the main source of infection. ${ }^{5,6}$ Furthermore, the incidence of leprosy has not fallen dramatically in many endemic countries, despite effective treatment of most lepromatous patients at an early stage of their disease. ${ }^{2}$ While early cases of multibacillary or paucibacillary disease may provide the bulk of excreted bacilli in some populations, in others self-healing infections which do not result in disease, but do have a transient period of nasal excretion, could be of great importance. ${ }^{4,5,9}$ A case of borderline-tuberculoid leprosy with a 'lepromatous' nodule in the nose ${ }^{10}$ might be an example of persistence of an initial bacilliferous lesion. The association of household contact with increased risk of disease ${ }^{11,12}$ and an antibody response ${ }^{5,8,13,14}$ suggests that direct spread of $M$. leprae from one infected individual to another may be important, although this could also occur by an indirect route as M. leprae can survive in nasal secretions. ${ }^{15}$ In one study, M. leprae has been found in the soil around the houses of leprosy patients by mouse footpad culture. ${ }^{16}$ There is also evidence that M. leprae may remain viable for some time, ${ }^{15,17}$ so it is possible that secreted bacilli in house dust could be a source of infection.

Most new patients have not had contact with leprosy patients who are shedding bacilli from skin lesions, but a reappraisal of Pedley's work on skin-skin transmission ${ }^{18,19}$ using PCR detection methods ${ }^{20}$ seems appropriate to confirm this work which used tinctorial staining methods to assess shedding of bacilli.

\section{Primary infection and immunity}

The route of entry of $M$. leprae to the body is still controversial, but the primary lesion of leprosy is still thought to be in the nose. ${ }^{21,22}$ There is little evidence for implantation of M. leprae into cuts or abrasions, ${ }^{23,24}$ or of transmission by blood-sucking insects. ${ }^{21,24,25}$ While occasional animals, notably armadillos and primates, can be infected by $M$. leprae, there is no evidence that they are a major source of infection within the population. However, subcutaneous infection of nine-banded armadillos does result in disseminated infection, ${ }^{24}$ albeit using high doses of viable $M$. leprae. While such mechanisms seem unlikely to account for large numbers of patients, they cannot be totally excluded and their validity should be further tested. ${ }^{23}$ For instance, the idea of transmission by blood-sucking insects is unproven, but testable by both experimental and PCR survey methods.

Large numbers of leprosy bacilli are excreted from the nose in untreated lepromatous leprosy. ${ }^{26-28}$ The nasal lesions in indeterminate cases can be bacilliferous ${ }^{29}$ and nasal excretion of leprosy bacilli is much more common than from any other part of the body in leprosy patients. ${ }^{21,30}$ It is therefore possible to postulate a hypothesis for early leprosy in which contact with $M$. leprae leads to primary nasal infection (Figure 1). This may be facilitated by nasal abrasions: infection may require the coincidence of infectious bacilli with an abrasion or other pathology of the nasal mucosa. ${ }^{5}$ In this model, haematogenous spread to skin and nerves would lead to the eventual development of clinical leprosy (Figure 1). A similar pattern of haematogenous spread has been observed in the nine-banded armadillo, 


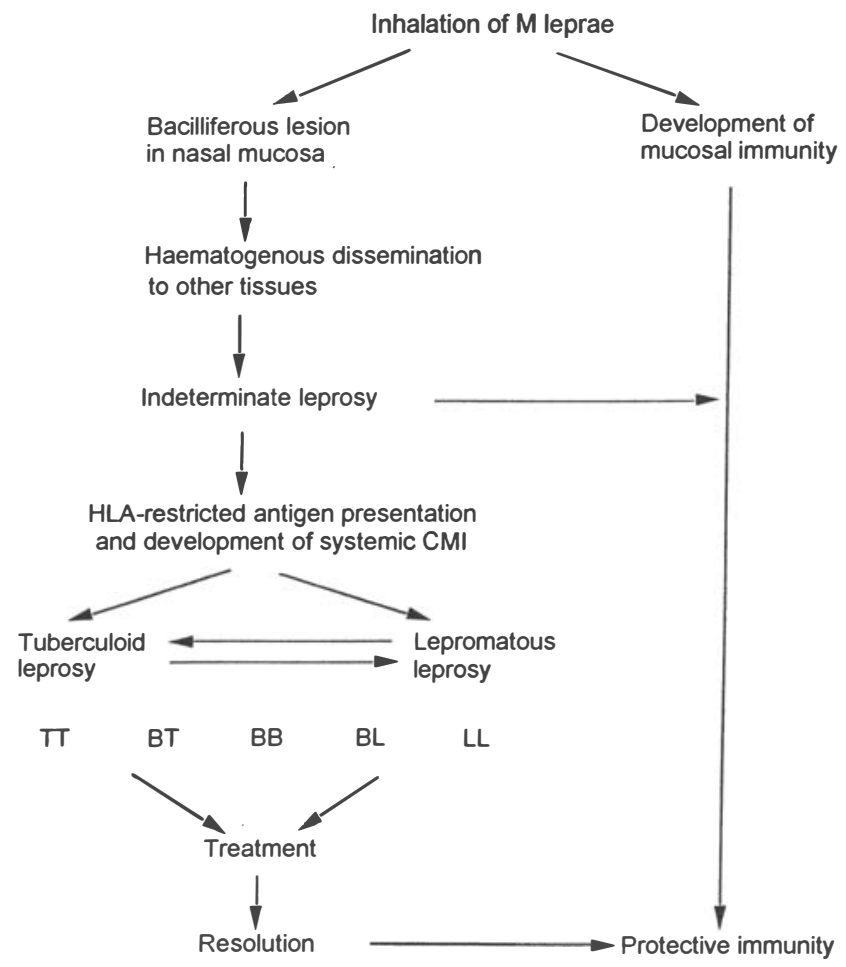

Figure 1. A model for the pathogenesis of leprosy.

although this was following primary subcutaneous inoculation, ${ }^{24}$ and in thymectomized mice following nasal instillation of M. leprae. ${ }^{31}$ Healing of the primary infection would accompany development of protective immunity and seroconversion. ${ }^{5,9}$

If this is correct, frequency of contact with a source of infection would be important in transmission. A large number of studies have shown the risk associated with household contact using both epidemiological and laboratory methods. ${ }^{5,1-13}$ However, it is possible that the primary nasal lesion goes through a bacilliferous phase in many infected individuals, accounting for the presence of $M$. leprae DNA in the nasal secretions of those living in endemic areas who have no known contact with leprosy. ${ }^{3,4,9}$ It is worth noting that PCR methods for the detection of $M$. leprae require the presence of DNA from at least five bacilli to give a positive result, ${ }^{20}$ and it is therefore likely that PCR positivity reflects the presence of substantial numbers of $M$. leprae in the nose. However, PCR positive individuals have not yet been subjected to detailed ENT examination to determine whether they have bacilliferous lesions or not. It is therefore theoretically possible that nasal excretion of $M$. leprae by subclinically infected individuals could be responsible for transmission, but this is by no means proven.

How does the primary lesion heal? The course of the infection (Figure 1) may depend upon the timing of the development of local mucosal immunity. There is evidence that the timing of development of cell-mediated immunity affects the development of indeterminate leprosy. ${ }^{32}$ The timing of development of effective immune responses does influence the progression of other infections by intracellular organisms: similar situations occur in the lung 
in primary tuberculosis ${ }^{7,33,34}$, in Buruli ulcer ${ }^{35}$ and in leishmaniasis. ${ }^{36}$ It is generally accepted that protection against leprosy and other intracellular infections requires cell-mediated immune responses. Successful immune defence against an initial $M$. leprae infection requires both prevention of dissemination and healing of the primary lesion, and there can be little doubt that an effective cell-mediated immune response could accomplish this. ${ }^{14,37}$ The development of cell-mediated responses in the nose is difficult to study, but unlike the systemic immune system, mucosal cell-mediated and humoral immunity may develop concommitantly. ${ }^{38,39}$ Since measurement of $\operatorname{IgA}$ responses is considerably easier, and all mucosal sites are linked by lymphocyte recirculation, ${ }^{38}$ it is possible to measure salivary antibody responses to $M$. leprae as a marker of anti-M. leprae immunity. Furthermore, mucosal $\operatorname{IgA}$ itself is of interest, since it mediates reduction of adherence of bacilli and opsonization responses which might be protective. ${ }^{14}$

\section{The mucosal immunology of leprosy}

Early and seminal studies by Abe et al. ${ }^{40-42}$ using the FLA-ABS test showed that most lepromatous patients do not have a salivary anti-M. leprae IgA (ML-IgA) response and that treated patients are more likely to have an ML-IgA response than untreated patients. The same group showed that a high proportion of contacts of leprosy patients have detectable MLIgA responses which they hoped might have diagnostic value. In this they were disappointed, but at the same time, we and others were becoming disillusioned with the overconcentration of studies on patients, who have demonstrably failed immunologically by getting leprosy. Initial studies in Bangladesh (253 subjects) and Fiji (163 subjects) showed that the ML-IgA response was least likely to be found in those with untreated leprosy or increased risk (i.e. household contacts), suggesting that, "the mucosal immune system might be of importance in a putative protective response to infection.... ${ }^{14}$ We were able to confirm that treated patients were much more likely to have a response than untreated patients, and found the highest numbers of ML-IgA positive individuals amongst hospital workers, ${ }^{14}$ who rarely get leprosy. Subsequent studies showed evidence of an IgA anti-LAM response in many individuals. $^{43}$

The development of PCR detection of putative early nasal infection ${ }^{20}$ gave us the possibility of testing our ideas further. In the MILEP1 study (304 subjects) in Miraj, Maharashtra, we concentrated our efforts on understanding the mucosal immune response to M. leprae in defined groups of patients, contacts and control subjects. ${ }^{9,37}$ The results of testing for salivary IgA directed against whole $M$. leprae can be interpreted in conjunction with the PCR results (putative infection/excretion of $M$. leprae) to provide a framework for understanding the pathogenesis of primary infection ${ }^{9}$ (Table 1). Non-exposed individuals are negative for salivary anti- $M$. leprae $\operatorname{IgA}\left(\mathrm{ML}^{-\operatorname{IgA}^{-}}\right)^{14,37}$ and do not show an amnestic response to challenge with nasal leprosin $A .{ }^{37}$ Nasal swabs are of course PCR negative $\left(\mathrm{PCR}^{-}\right)$. On contact with $M$. leprae, non-exposed individuals are likely to acquire a primary infection. This will be transient in most individuals, but as a result their nasal excretions may contain $M$. leprae, making them $\mathrm{PCR}$ positive $\left(\mathrm{PCR}^{+}\right)$. At an undetermined and probably variable time after infection, immunity develops leading to $\operatorname{IgA}$ positivity $\left(\mathrm{ML}_{\mathrm{IgA}}{ }^{+}\right.$). Development of a mucosal immune response is more rapid than healing of this initial lesion resulting in $\mathrm{PCR}^{+} / \mathrm{ML}-\mathrm{IgA}^{+}$individuals being as common $(6 / 204)$ as $\mathrm{PCR}+/$ $\mathrm{MIL}^{-} \operatorname{IgA}{ }^{-}$individuals (7/204). ${ }^{9} \mathrm{PCR}^{+}$individuals become $\mathrm{PCR}^{-}$within a year, ${ }^{4,9}$ but 
Table 1. PCR and mucosal immunity during the pathogenesis of early leprosy infection

\begin{tabular}{lll}
\hline & & \\
1. & Non-exposed & IgA-PCR- \\
2. & 1st exposure & IgA- PCR+ \\
3. & Resolving infection & IgA + PCR + \\
4. & Immune (?protected) & IgA + PCR - \\
\hline
\end{tabular}

the $\operatorname{IgA}$ response is much longer lasting. ${ }^{9,37}$ Although salivary $\operatorname{IgA}$ anti-M. leprae antibody may be absent after a year or so, the capability to produce such a response can be detected by mucosal challenge testing up to 10 years following exposure to M. leprae. ${ }^{37}$ Other markers of infection such as serum PGL1 IgM antibody levels show a similar pattern within the population, ${ }^{5,9,13}$ but correlate poorly with PCR. ${ }^{5}$ It is possible that serum antibody levels rise as PCR positivity declines and that the correlation between these two markers is therefore displaced in time.

The most worrying result of the MILEP1 study was the low level of ML-IgA positivity (33\%) amongst a group of 58 'control' subjects with no known leprosy contact in comparison with previous studies using the same ELISA protocol in Fiji and Bangladesh (79\% and $69 \%$, respectively ${ }^{14}$ ), despite the continuing presence of nasal PCR positivity suggestive of infection in the community. ${ }^{9}$ The numbers are small, but suggest that there may have been a decrease in immunity in the general population in this area while transmission continues, particularly amongst household contacts of leprosy patients. ${ }^{9}$ The reasons for this decline, if it is a real phenomenon, are not clear. However, this area has had an effective MDT control programme for 12 years, whereas the previous study was carried out in the pre-MDT era.

The data from the MILEP1 study, ${ }^{9,37}$ support our hypothesis that mucosal immunity develops during resolution of presumed infection and that it may be related to the development of protective immunity. Since long-term immunity is probably determined by the outcome of initial infection long before clinical leprosy develops, there is a need to investigate the pathogenesis of the primary lesion more thoroughly. We have now embarked on a much larger study (MILEP2) in which PCR and IgA studies are being performed in whole villages near Miraj and in Ethiopia: some 1200 subjects have been examined to date with similar results to those obtained previously (unpublished data). These whole population surveys will define transmission and mucosal immunity within a well-defined epidemiological framework, allowing a strategy foreradication to be developed. Similar studies are taking place in Indonesia (Klatser, personal communication).

\section{Breaking the cycle of transmission}

The control of infectious disease requires control of transmission. Since leprosy has such a long incubation period during which the infection can be passed on, development of clinical disease is a poor marker of infection. This makes study of the process of transmission and development of protective immunity difficult, but knowledge of these processes is essential for the design of effective intervention in endemic countries.

We suggest that the key to effective control is the recognition that leprosy infection 
occurs in clusters, as do many other infectious diseases. ${ }^{4}$ Treatment of all potentially infected individuals in such clusters could be a powerful adjunct to MDT control and might well lead to eradication of the disease. However, PCR requires sophisticated facilities and is unlikely to form part of a practical detection system for field use. Since few of the infected individuals are likely to develop disease and there is already an MDT programme in place, alternative (albeit less specific methods) to identify clusters are appropriate, since all individuals in a cluster could receive single dose therapy with mycobactericidal drugs such as rifampicin or ofloxacin (probably in combination to avoid the theoretical risk of resistance despite the increased risk of toxicity this would entail). Alternatives which may be good enough indicators of recent infection to achieve community-oriented control may include PGL1 serology, ${ }^{13}$ which needs to be re-evaluated in the light of studies of the temporal pathogenesis of the primary infection.

Where does first exposure occur? One can only speculate at present. For contacts, the home is probably most important, while for others exposure in childhood may well occur when they start to attend school. ${ }^{11,44}$ Public places such as tea houses, buses, factories and hospitals are possibly less likely to be important sources of infection, since in most leprosy endemic populations, individuals will have met $M$. leprae before they encounter these sites and contact may be transient. ${ }^{44,45}$ There is of course likely to be variation between communities, and PCR or antigen detection studies to determine where M. leprae can be found in the environment would be valuable. However, in many communities, we believe that intervention (drug or vaccine) in school and home might interrupt transmission sufficiently to eradicate infection. The MILEP2 and related studies should provide answers to some of these questions. The finding of reduced levels of immunity in adults with no history of leprosy exposure within a leprosy control area ${ }^{9}$ supports the idea that childhood exposure is important and it has long been known that childhood disease becomes rare in societies from which leprosy is disappearing. ${ }^{46}$ Understanding of the source of infection and pattern of transmission should allow the design of epidemiological methods to delineate clusters using minimal PCR or serological testing in highly endemic areas.

In populations where numbers of immune individuals decline to about $10 \%$ of the population, a screen for immunity may be more appropriate. This would have similarities with the use of Mantoux testing for contact tracing of tuberculosis suspects in areas of low endemicity. Salivary ML-IgA response ${ }^{9}$ and skin testing ${ }^{7}$ seem the most obvious alternatives. The former has many advantages since it is objective, non-invasive, and can be automated or simplified (development of a dipstick test may well be feasible). There is every chance that it will be possible to identify clusters of at risk individuals. This has considerable implications for the choice of immunoprophylaxis or chemoprophylaxis.

\section{Immunoprophylaxis or chemoprophylaxis?}

Rational vaccine design requires knowledge of the protective immune mechanism, which must be shown to confer protection as long as immunity persists. This allows potential vaccines to be tested to ensure that they produce the correct response in unexposed individuals before large vaccine trials are attempted. A vaccine meant for whole population protection may not be required in leprosy if a strategy of targeting recent infection is followed: secondary immunoprophylaxis (i.e. immunotherapy of infection before disease occurs) would be sufficient. ${ }^{47}$ Despite the large amount of resources devoted to finding a 
leprosy vaccine, there is at present no vaccine which fulfils these requirements and is available for widespread use, with the possible exception of BCG in some areas. ${ }^{47,48}$

The other option is chemoprophylaxis. The recognition that contacts of leprosy patients were at increased risk of contracting the disease led to a number of trials of chemoprophylaxis of leprosy using long-term dapsone treatment. No distinction was possible between primary chemoprophylaxis (protection of uninfected individuals) and secondary chemoprophylaxis (treatment of presumed infection to prevent the development of clinical disease). The results of these studies varied, initially with some encouraging results, ${ }^{49-51}$ but controlled trials at Chingleput of this approach were judged less satisfactory ${ }^{52,53}$ despite $50 \%$ protection levels. There are clearly problems in giving long-term treatment to children with potentially toxic drugs, although at the time the even more extreme measure of separating children of leprosy patients from their parents was commonplace. ${ }^{49}$ The results of childhood chemoprophylaxis encouraged Sloan and co-workers ${ }^{54-57}$ to attempt whole population chemoprophylaxis in Micronesia (Pingelap atoll), where the incidence was estimated at $7 / 1000$ per annum and the prevalence $6.6 \%$. Lepromin positivity increased with age from $29 \%$ at $<4$ years, $61 \%$ in the 5-9 age group, $86 \%$ in the $10-14$ age group, to $100 \%$ in the 15-19 age group. This suggests widespread early childhood exposure to $M$. leprae. A depot preparation of dapsone (Acedapsone or DADDS) was offered to the whole population and actually given to $51 \%$ for 3 years (1967-69) at 3-monthly intervals. However, every eligible individual in the population received at least one injection. Six new cases occured during the phase of DADDS administration, but repeated examination in 1969 and 1970 revealed no new cases. The authors ${ }^{55}$ concluded, '...the next logical step would be a series of experiments to test how far one must extend DADDS chemoprophylaxis into the web of household and neighbourhood contacts before one runs into serious problems of noncooperation and/or inefficient benefit.' Despite the success of their approach, this point was largely ignored. ${ }^{54,55}$ However, such isolated communities are rare and the problems of giving such long-term treatment as well as the cost lead to a preference for immunoprophylaxis over the next 20 years.

In recent years, chemoprophylaxis has been tried in another isolated community in the Southern Marquesas using a single dose of $25 \mathrm{mg} / \mathrm{kg}$ rifampicin given to $98.7 \%$ of the population. ${ }^{58}$ The rapid occurence of a skin lesion in a boy 3 months after chemoprophylaxis seems to have worried the authors, although this patient may well have been incubating the disease for some time before prophylaxis. A further case was detected at 21 months post-prophylaxis. ${ }^{59}$ The authors concluded that their chemoprophylaxis was responsible for $50 \%$ protection, but that the costs involved in whole population treatment made chemoprophylaxis unsuitable for leprosy control. ${ }^{59}$ The current WHO study of single dose treatment using a combination of ofloxacin, minocycline and rifampicin for single lesion leprosy is based on the premise that there are few bacilli to kill in single lesions and is proving clinically acceptable (V. J. Edward, personal communication). Such multi-agent single dose therapy might be more suitable for chemoprophylaxis. Nasal lesions may be bacilliferous, but they can be quite small ${ }^{26,27}$ and single dose therapy with a cidal drug regimen might well result in more rapid healing with development of immunity.

We believe that drug-based intervention studies designed to interrupt transmission of infection rather than to prevent development of the disease might add to the efficacy of MDT programmes. The impact of MDT control programmes on leprosy transmission is largely unknown. However, the likely effect of stopping/reducing such programmes where the prevalence of leprosy has fallen to low levels can be predicted from the evidence of 
continuing transmission even in good MDT control programmes ${ }^{9}$ and the results of the acedapsone trial in Pingelap ${ }^{54-57}$ - leprosy would slowly return. Maintenance of current MDT programmes is financially difficult, but currently available evidence suggests that improving their efficacy might allow eradication.

\section{Conclusion}

Since there is no large animal or environmental reservoir of infection, eradication of leprosy is feasible. Adoption of a strategy for control based on the detection of clusters of infection needs more information, but the prospect is an exciting one. Few ENT surgeons have taken an interest in leprosy since the studies by Rex Barton in the 1970s defined the nasal lesions ${ }^{10,26,27}$ and there is a general lack of information about the pathogenesis of primary infection which derives in part from the concentration of effort on established disease. Studies of transmission have been unjustifiably neglected for far too long. ${ }^{2,60}$ Maintenance of control programmes is expensive and becomes increasingly more difficult to sustain as numbers of cases on treatment fall. Eradication may prove a more attainable long term objective than elimination, but the time to develop the tools is now, while the MDT programmes are still in place.

\section{Acknowledgements}

The development of this work has benefited from many useful discussions, particularly with partners in the MILEP projects: Dr P. D. Samson, Dr V. K. Edward, Dr P. R. Klatser, Dr W. Terpstra, Professor N. K. Mehra, Dr M. Giphart, Dr J. J. Ryon and Professor R. R. P. de Vries. We are grateful to the Commission of the European Communities, Directorate General XII for Science, Research and Development (TS*CT92*0062 and IC18CT960047) for their support.

\section{References}

1 World Health Organisation. Progress towards the elimination of leprosy as a public health problem. Weekly Epidemiol Rec, 1996; 71: 149-156.

2 Editorial. Leprosy beyond the year 2000. Lancet, 1998; 350: 1717.

3 Klatser PR, van Beers S, Madjid B, Day R, de Wit MYL. Detection of Mycobacterium leprae nasal carriage in a leprosy endemic population. J Clin Microbiol, 1993; 31: 2947.

${ }^{4}$ Hatta H, van Beers SM, Madjid B, de Wit MYL, Klatser PR. Distribution and persistence of Mycobacterium leprae nasal carriage among a population in which leprosy is endemic in Indonesia. Trans R Soc Trop Med Hyg, 1995; 89: 381-385.

5 van Beers SM, de Wit MYL, Klatser PR. The epidemiology of Mycobacterium leprae: recent insight. FEMS Microbiol Lett, 1996; 136: 221-230.

6 Godal T, Negassi K. Subclinical infection in leprosy. Brit Med J, 1973; 3: 557-559.

${ }^{7}$ Cree IA, Smith WCS, Rees RJW, Beck JS. The influence of anti-mycobacterial chemotherapy on delayed hypersensitivity skin-test reactions in leprosy patients. Lepr Rev, 1988; 59: 145-151.

${ }^{8}$ Cree IA, Smith WCS, Beck JS. Serum antibody responses to mycobacteria in leprosy patients and their contacts. Lepr Rev, 1988; 59: 317-328.

9 Ramaprasad P, Fernando A, Madhale S, Rao JR, Edward VK, Samson PD, Klatser PR, de Wit MYL, Smith WCS, Cree IA. Transmission and protection in leprosy: indications of the role of mucosal immunity. Lepr Rev, 1997; 68: 301-315. 
${ }^{10}$ Barton RPE, Hogerzeil LM, McDougall AC. Borderline-tuberculoid leprosy with a lepromatous nodule of the nasal mucosa: a case report. Lepr India, 1980; 52: 114-118.

11 Rao PS, Karat AB, Kaliaperumal VG, Karat S. Transmission of leprosy within households. Int J Lepr, 1975; 43: 45-54.

12 Noordeen SK. The epidemiology of leprosy. In: Leprosy, RC Hastings, ed. Churchill Livingstone, Edinburgh. 1985: 15-30.

13 Baumgart KW, Britton WJ, Mullins RJ, Basten A, Barnetson RS. Subclinical infection with Mycobacterium leprae-a problem for leprosy control strategies. Trans $R$ Soc Trop Med Hyg, 1993; 87: 412-415.

${ }^{14}$ Cree IA, Sharpe S, Sturrock NDC, Cochrance IH, Dawlako EC, Smith WCS, Beck JS. Mucosal immunity to mycobacteria in leprosy patients and their contacts. Lepr Rev, 1988; 59: 309-316.

15 Davey TF, Rees RJW. The nasal discharge in leprosy; clinical and bacteriological aspects. Lepr Rev, 1974; 45: $121-134$

16 Kazda J, Ganapati R, Revankar C, Buchanan TM, Young DB, Irgens LM. Isolation of environment-derived Mycobacterium le prae from soil in Bombay. Lepr Rev, 1986; 57: 201-208.

17 Desikan KV, Sreevatsa. Extended studies on the viability of Mycobacterium leprae outside the human body. Lepr Rev, 1995; 66: 287-295.

18 Pedley JC. Composite contact smears: a method of demonstrating the non-emergence of Mycobacterium leprae from intact lepromatous skin. Lepr Rev, 1970; 41: 31-43.

19 Pedley JC. Summary of the results of a search of the skin surface for Mycobacterium leprae. Lepr Rev, 1970; 41: $167-168$.

20 de Wit MYL., Douglas JT, McFadden J, Klatser PR. Polymerase chain reaction for detection of Mycobacterium leprae in nasal swab specimens. J Clin Microbiol, 1993; 31: 502-506.

21 Pallen MJ, McDermott RD.How might Mycobacterium leprae enter the body? Lepr Rev, 1986; 57: 289.

22 McDermott-Lancaster RD, McDougall AC. Mode of transmission and histology of M. leprae infection in nude mice. Int J Exp Pathol, 1990; 71: 689-700.

23 Job CK, Baskaran B, Jayakumar J, Aschoff M. Histopathologic evidence to show that indeterminate leprosy may be a primary lesion of the disease. Int J Lepr, 1997; 65: 443-449.

${ }^{24}$ Job CK, Drain V, Truman R, Deming AT, Sanchez RM, Hastings RC. The pathogenesis of leprosy in the ninebanded armadillo and the significance of IgM antibodies to PGL-1. Ind J Lepr, 1992; 64: 137-151.

25 Narayanan E, Sreevatsa, Kirchheimer WF, Bedi BM. Transfer of leprosy bacilli from patients to mouse footpads by Aedes aegypti. Lepr Ind, 1977; 49: 181-186.

26 Barton RPE. A clinical study of the nose in lepromatous leprosy. Lepr Rev, 1974; 45: 135-144.

27 Barton RPE, Rees RJW, McDougall AC, Ellard GA. The nose in lepromatous leprosy: bacteriological and histological studies of patients treated with dapsone monotherapy for varying periods of time. Int J Lepr, 1979; 50: $58-67$.

28 Green CA, Katoch VM, Desikan KV. Quantitative estimation of Mycobacterium leprae in exhaled nasal breath. Lepr Rev, 1983; 54: 337-340.

29 Chacko CJG, Bhanu T, Victor V, Alexander R, Taylor PM, Job CK. The significance of changes in the nasal mucosa in indeterminate, tuberculoid and borderline leprosy. Lepr Ind, 1979; 51: 8-22.

30 Pedley JC. The nasal mucus in leprosy. Lepr Rev, 1973; 44: 33-35.

31 Rees RJW, McDougall AC, Weddell AGM. The nose in mice with experimental human leprosy. Lepr Rev, 1974; 45: $112-120$.

32 Myrvang B, Godal T, Feek CM, Ridley DS, Samuel DD. Immune response to Mycobacterium leprae in indeterminate leprosy patients. Acta Pathol Microbiol Scand [B], 1973; 81: 615-620.

33 Bjune G, Closs O, Barnetson RStC. Early events in the host-parasite relationship and immune response in clinical leprosy: its possible importance for leprosy control. Clin Exp Immunol, 1983; 54: 289-297.

${ }^{34}$ Lucas SB, Cree IA. Multisystem disease. In: Pathology, IA Cree, ed., Chapman \& Hall, London, 1997: $150-155$.

35 Grange JM. Mycobacteria and human disease. Edward Amold Publishers Ltd, London, 1988: 142-152.

${ }^{36}$ Reiner SL, Locksley RM. The regulation of immunity to Leishmania major. Annu Rev Immunol, 1995; 13: $151-177$.

37 Ramaprasad P, Cree IA, Oluwole M, Samson PD. Development of a mucosal challenge test for leprosy using leprosin A. J Immunol Methods, 1995; 188: 239-246.

38 Allardyce RA, Bienenstock J. The mucosal immune system in health and disease with an emphasis on parasitic infection. Bull WHO, 1984; 62: 7-25.

39 Arny M, Kelly-Hatfield P, Lamm ME, Phillips-Quagliata JM. T-cell help for the IgA response: the function of Tcells from different lymphoid organs in regulating the proportions of plasma cells expressing various isotypes. Cell Immunol, 1984; 89: 95-112.

40 Abe M, Minagawa F, Yoshino Y, Itoh M. Immunoglobulin levels and antibody titers in nasal secretions versus serum of leprosy patients. Int J Lepr 1974; 42: 508 (Abstract).

41 Abe M, Yoshino Y, Minagawa F, Miyaji I, Sampoonachot P, Ozawa T, Sakamoto Y, Saito T, Saikawa K. Salivary immunoglobulins and antibody activities in leprosy. Int J Lepr, 1984; 52: 343-350.

42 Abe M, Miyaji I, Okushita T, Minagawa F, Yoshino Y, Sakamoto Y, Saikawa K. Anti-mycobacterial antibodies in saliva. Lepr Rev, 1986; 57: 213-223. 
43 Bothamley GH, Cree IA. Antibody (IgA) specificity in leprosy. In: Advances in mucosal immunology (Proceedings of the Vth International Congress of Mucosal Immunology 1989), TT MacDonald, SJ Challacombe, PW Bland, CR Stokes, RV Heatley \& AM Mowat, eds, Kluwer Academic Publishers, Dordrecht, Netherlands, 1990: 493-494.

${ }^{44}$ Huang-CL. The transmission of leprosy in man. Int J Lepr, 1980; 48: 309-318.

45 Davey TF. A day in the life of Yeeranna-a cautionary tale. Lepr Rev, 1978; 49: 269-274.

${ }^{46}$ Irgens LM. Leprosy in Norway-an epidemiological study based on a national patient registry. Lepr Rev, 1980; 51: $1-130$.

${ }^{47}$ Convit J, Sampson C, Zuniga M, Smith PG, Plata J, Silva J, Molina J, Pinardi ME, Bloom BR, Salgado A. Immunoprophylactic trial with combined Mycobacterium leprae/BCG vaccine against leprosy: preliminary results. Lancet, 1992; 339: 446-450.

48 Karonga Prevention Trial Group. Randomised controlled trial of single BCG, repeated BCG, or combined BCG and killed Mycobacterium leprae vaccine for prevention of leprosy and tuberculosis in Malawi. Lancet, 1996; 348: 17-24.

49 Brechet RA. Chemoprophylaxis with DDS, mainly in children: a short trial. Lepr Rev, 1965; 36: 143-144.

${ }^{50}$ Guinto RS. Chemoprophylaxis and BCG vaccination against leprosy. Int J Lepr, 1966; 34: 313-316.

51 Long ER. The chemoprophylaxis of mycobacterial diseases. Int J Lepr, 1966; 34: 65-67.

52 Noordeen SK, Neelan PN. Value of dapsone as a chemoprophylactic agent against leprosy (abstract IA8). In: Proceedings of the XI International Leprosy Congress, Mexico City, 1984: 4.

53 Neelan PN, Sirumban P, Sivaprasad N. Limited duration acedapsone prophylaxis in leprosy. Ind J Lepr, 1986; 58: $251-256$

54 Sloan NR, Worth RM, Jano B, Fasal P, Shephard CC. Repository acedapsone in leprosy chemoprophylaxis and treatment. Lancet, 1971; 2: 525-526.

55 Sloan NR, Worth RM, Jano B, Fasal P, Shepard CC. Acedapsone in leprosy chemoprophylaxis: field trial in three high-prevalence villages in Micronesia. Int J Lepr, 1972; 40: 40-47.

56 World Health Organisation. Strategy of leprosy control. WHO Chronicle, 1978; 32: 193-199.

57 Russell DA, Worth RM, Jano B, Fasal P, Shephard CC. Acedapsone in the prevention of leprosy: field trial in three high prevalence villages in Micronesia. Am J Trop Med Hyg, 1979; 28: 559-563.

58 Cartel JL, Chanteau S, Boutin JP, Taylor R, Plichart R, Roux J, Celerier P, Grosset JH. Implementation of chemoprophylaxis of leprosy in the Southern Marquesas with a single dose of $25 \mathrm{mg}$ per kg rifampin. Int J Lepr, 1989; 57: 810-816

59 Cartel JL, Chanteau S, Glaziou P, Roux JF, Grosset J. Five year evaluation of a program of chemoprophylaxis for leprosy using a single dose of $25 \mathrm{mg} / \mathrm{kg}$ rifampicin. Int J Lepr, 1993; 61: 61A.

60 John, TJ. Leprosy in the year 2000. Lancet, 1998; 351: 756. 